\title{
Real-time Laboratory-Based Influenza Surveillance with Xpert Flu
}

\author{
Andrea Dugas ${ }^{\star 1}$, Howard Burkom ${ }^{2}$, Richard Rothman ${ }^{1}$ and on behalf of the National \\ Emergency Department Influenza Consortium ${ }^{1}$
}

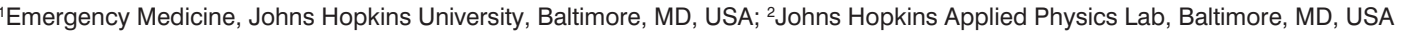

\section{Objective}

To demonstrate the feasibility and validity of a novel electronic surveillance system utilizing a cloud-based interface that consolidates laboratory test results and geographical information in real-time.

\section{Introduction}

Previous studies have demonstrated the benefit of laboratory surveillance and its capability to accurately detect influenza outbreaks earlier than syndromic surveillance ${ }^{1,2}$. Current laboratory surveillance has an approximate 2-week lag due to laboratory test turn-around time and data collection ${ }^{3}$. In order to provide real-time access to aggregated test results, we utilized direct cloud connectivity with a rapid PCR-based influenza test, Xpert Flu, to centrally consolidate test results along with GIS data. On-site, type-specific results were available to physicians and uploaded for public health awareness within 100 minutes of patient nasopharyngeal swab.

\section{Methods}

We implemented a real-time laboratory-based influenza surveillance system at two academic emergency medical departments. From November 2013 - April 2014 we systematically enrolled and tested patients with an acute respiratory illness using Cepheid Xpert Flu, a rapid highly sensitive PCR-based assay that provides significant improvement over traditional rapid antigen tests ${ }^{4}$. Test results were instantaneously uploaded to a cloud-based data aggregation system including the test result, time and date, and geolocation.

\section{Results}

From November 2013 - April 2014, the cloud-based laboratory surveillance system automatically uploaded test results and associated data which were immediately available to affiliated clinicians and local, state, and national public health end users. A total of 1032 subjects were tested and 82 cases of influenza were identified as displayed in Figure 1. Results from these two EDs differentiated in real time the regional epidemic curves and showed the type-A virus wave followed by the smaller type-B virus wave.

\section{Conclusions}

This novel, real-time laboratory-based surveillance system automatically uploaded and aggregated flu test results and the associated times and locations. Expansion of this real-time capability to sentinel facilities could improve both local and national surveillance and response, reducing the need for syndromic influenza surveillance. Patient demographic detail will be added to real-time flu results in the upcoming season for enriched public health utility.

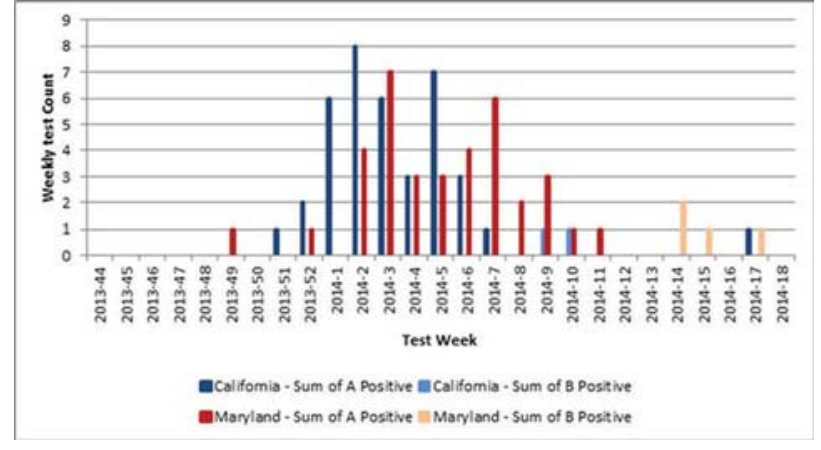

Figure 1: Influenza test results by Week throughout the 2013-2014 Influenza season

\section{Keywords}

Influenza; Clinical Decision Support; Surveillance

\section{Acknowledgments}

This work was supported by the Cooperative Agreement IDSEP13001401-00 from The Assistant Secretary for Preparedness and Response. Its contents are solely the responsibility of the authors and do not necessarily represent the official views of The Assistant Secretary for Preparedness and Response (within the U.S. Department of Health and Human Services).

\section{References}

1. Baumbach J, Mueller M, Smelser C, Albanese B, Sewell CM. Enhancement of influenza surveillance with aggregate rapid influenza test results: New Mexico, 2003-2007. Am J Public Health. 2009;99(Suppl 2):S372-377.

2. Ghosh TS, Vogt RL. Active influenza surveillance at the local level: a model for local health agencies. Am J Public Health. 2008;98(2):1315.

3. Centers for Disease Control and Prevention. The National Respiratory and Enteric Virus Surveillance System (NREVSS). 2011; http://www. cdc.gov/surveillance/nrevss/. Accessed Nov 25, 2011.

4. DiMaio MA, Sahoo MK, Waggoner J, Pinsky BA. Comparison of Xpert Flu rapid nucleic acid testing with rapid antigen testing for the diagnosis of influenza A and B. J Virol Methods. 2012;186(1-2):137140.

\footnotetext{
*Andrea Dugas

E-mail: adugas1@jhmi.edu
} 